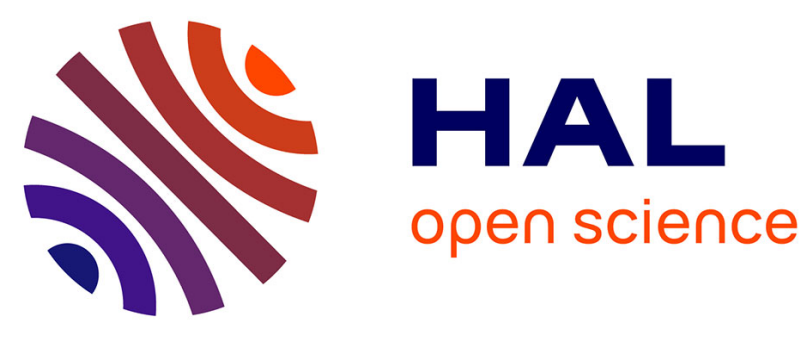

\title{
From Chains to Monolayers: Nanoparticle Assembly Driven by Smectic Topological Defects
}

Syou P'Heng Do, Missaoui Amine, Alessandro Coati, Delphine Coursault, Haïfa Jeridi, Andrea Resta, Nicolas Goubet, Michal Maksymilian Wojcik, Arnaud Choux, Sebastien Royer, et al.

\section{To cite this version:}

Syou P'Heng Do, Missaoui Amine, Alessandro Coati, Delphine Coursault, Haïfa Jeridi, et al.. From Chains to Monolayers: Nanoparticle Assembly Driven by Smectic Topological Defects. Nano Letters, 2020, 20 (3), pp.1598-1606. 10.1021/acs.nanolett.9b04347 . hal-02450544

\author{
HAL Id: hal-02450544 \\ https://hal.science/hal-02450544
}

Submitted on 15 Dec 2020

HAL is a multi-disciplinary open access archive for the deposit and dissemination of scientific research documents, whether they are published or not. The documents may come from teaching and research institutions in France or abroad, or from public or private research centers.
L'archive ouverte pluridisciplinaire $\mathbf{H A L}$, est destinée au dépôt et à la diffusion de documents scientifiques de niveau recherche, publiés ou non, émanant des établissements d'enseignement et de recherche français ou étrangers, des laboratoires publics ou privés. 


\section{From Chains to Monolayers : Nanoparticle Assembly Driven by Smectic Topological Defects}

Syou-P’heng Do, ${ }^{\dagger}$ Amine Missaoui, ${ }^{\dagger}$ Alessandro Coati, ${ }^{\ddagger}$ Delphine Coursault,,${ }^{\dagger} \uparrow$ Haifa Jeridi, ${ }^{\dagger}$ Andrea Resta, ${ }^{\ddagger}$ Nicolas Goubet, ${ }^{\dagger}$ Michal M. Wojcik, ${ }^{\S}$ Arnaud Choux, ${ }^{\dagger}$ Sébastien Royer, ${ }^{\dagger}$ Emrick Briand, ${ }^{\dagger}$ Bertrand Donnio, $\|$ Jean Louis

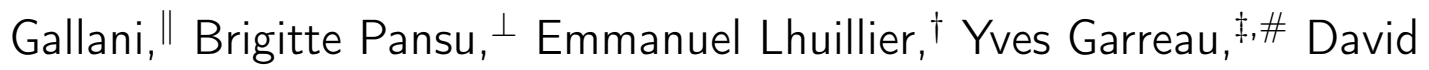
Babonneau, ${ }^{@}$ Michel Goldmann, ${ }^{\dagger, \ddagger}$ Doru Constantin, ${ }^{\perp}$ Bruno Gallas, ${ }^{\dagger}$ Bernard Croset, $^{\dagger}$ and Emmanuelle Lacaze ${ }^{*, \dagger}$

$\dagger$ †orbonne Université, Faculté des Sciences, CNRS, Institut des Nano-Sciences de Paris (INSP), 4 pl Jussieu 75005 PARIS, France

$\ddagger$ Synchrotron Soleil, BP 48, L’Orme des Merisiers, 91192 Gif sur Yvette Cedex, France

ๆUniversité de Bordeaux, CNRS, LOMA, UMR 5798, Talence F-33400, France

$\S$ Faculty of Chemistry, University of Warsaw, Pasteura 1, 02-093 Warsaw, Poland || Institut de Physique et de Chimie des Matériaux de Strasbourg (IPCMS), UMR 7504, CNRS-Université de Strasbourg, BP 43, 23 rue du Loess, F-67034 Strasbourg Cedex 2,

France

$\perp$ Laboratoire de Physique des Solides, Bat. 510, UMR-CNRS 8502, Université Paris-Sud, Université Paris-Saclay, F-91405, Orsay, France

\#Université de Paris, Laboratoire Matériaux et Phénomènes Quantiques, CNRS, F-75013, Paris, France

@Institut Pprime, Département Physique et Mécanique des Matériaux, UPR 3346 CNRS, Université de Poitiers, SP2MI,TSA 41123, 86073 Poitiers Cedex 9, France

E-mail: emmanuelle.2acaze@insp.jussieu.fr 


\begin{abstract}
In this article, we show how advanced hierarchical structures of topological defects in the so-called smectic oily streaks can be used to sequentially transfer their geometrical features to gold nanospheres. We use two kinds of topological defects, 1D dislocations and $2 \mathrm{D}$ ribbon-like topological defects. The large trapping efficiency of the smectic dislocation cores not only surpasses that of the elastically distorted zones around the cores but also the one of the $2 \mathrm{D}$ ribbon-like topological defect. This enables the formation of a large number of aligned NP chains, within the dislocation cores that can be quasi-fully filled without any significant aggregation outside the cores. When the NP concentration is large enough to entirely fill the dislocation cores, the LC confinement varies from $1 \mathrm{D}$ to $2 \mathrm{D}$. We demonstrate that the $2 \mathrm{D}$ topological defect cores induce a confinement that leads to planar hexagonal networks of NPs. We then draw the phase diagram driven by NP concentration, associated with the sequential confinements induced by these two kinds of topological defects. Owing to the excellent large-scale order of these defect cores, not only the NP chains but also the NP hexagonal networks can be oriented along the desired direction, suggesting a possible new route for the creation of either 1D or 2D highly anisotropic NP networks. In addition, these results open rich perspectives based on the possible creation of coexisting NP assemblies of different kinds, localized in different confining areas of a same smectic film that would thus interact thanks to their proximity but also would interact via the surrounding soft matter matrix.
\end{abstract}

Keywords: Smectic, Topological defects, Oily Streaks, Nanoparticles, gold, X-ray, dislocations, assembly 
Liquid crystal (LC) topological defects are fascinating objects, which are actively studied nowadays as model systems for a deep understanding of topological matter ${ }^{1-4}$.It has been also discovered that they can attract and trap inorganic nanoparticles (NPs). The trapping of NPs within topological defect cores allows for the release of the defect core energy and for the stabilization of the composite systems ${ }^{5-9}$. LC topological defects may thus be useful as a matrix for new kinds of NP organizations templated by the defect geometry ${ }^{10-12}$. The research on LC topological defects is constantly expanding and many attractive systems are emerging, in particular characterized by the formation of different kinds of topological defects in a single system, like LC droplets or shells ${ }^{13-16}$ or even oriented thin smectic films ${ }^{17,18}$. Our aim is thus to go beyond the demonstration of the trapping phenomenon of NPs by LC topological defects and to understand the specificity of each topological defect regarding their NP templating property. In particular, can we use hierarchical structures of topological defects in LC materials to hierarchically build composites made of different kinds of NP assemblies?

To answer this question, we have used smectic oily streaks made of an array of oriented topological defects, smectic dislocations (1D topological defect), together with ribbon-like grain boundaries (2D topological defect) ${ }^{17}$. We have increased the concentration of gold NPs whose Localized Surface Plasmon (LSP) resonance in the smectic oily streaks is followed as a function of the local concentration. By combining spectrophotometry, Rutherford Back Scattering (RBS) measurements and X-ray diffraction at the Soleil synchrotron facility, we bring evidence for two different confinement features for the gold NPs. A 1D confinement starts first, where NP chains are formed until the dislocations are quasi-fully filled by the NPs, followed by a 2D confinement at higher concentration. We demonstrate that the 2D confinement induced by 2D topological defects leads to formation of planar oriented hexagonal networks of NPs $(\langle 10\rangle$ direction more favorably oriented parallel to the LC defect orientation). Our results also highlight how a hierarchical structure of topological defects can be used to sequentially build different kinds of NP assemblies, in relation with different kind 
of induced confinement by each topological defect. Ultimately this leads to the coexistence of 1D and 2D NP assemblies at different localizations in the same film, opening rich perspectives for future composites formed within LC systems made of different kinds of topological defects.
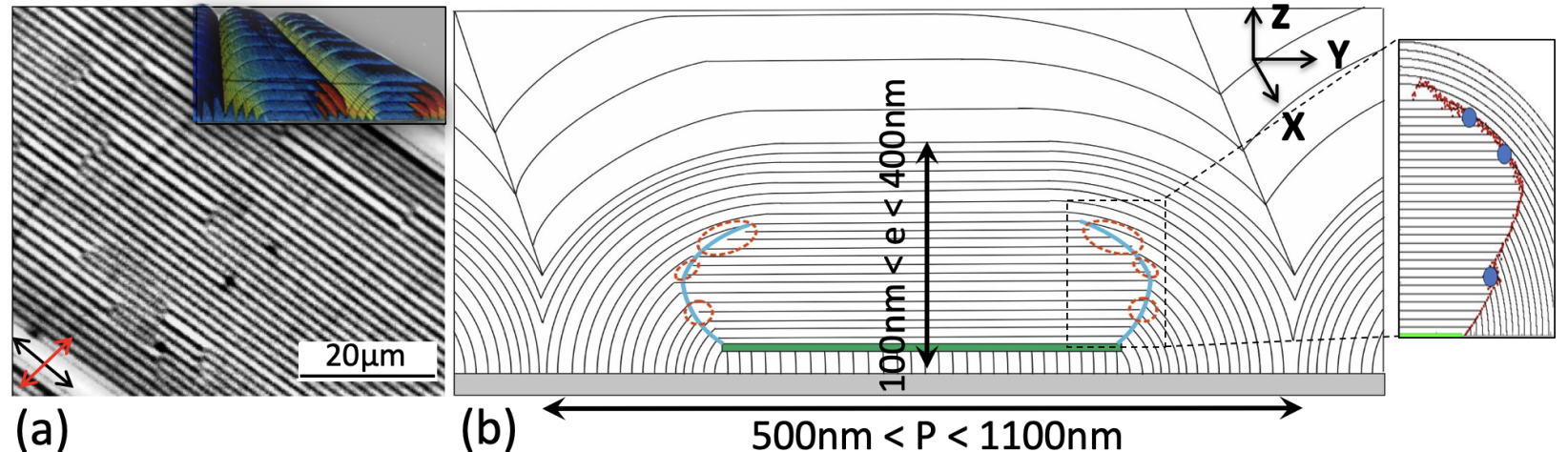

Figure 1: (a) Polarized optical microscopy image of oily streaks observed from the top of a $150 \mathrm{~nm}$-thick sample. Each stripe along OX corresponds to one hemicylinder and two hemicylinders in perspective are represented (b) Detail in side-view (in the (Y, Z) plane) of the smectic layers stacked in one flattened hemicylinder with two rotating grain boundaries (RGB) in red, including dispersed edge dislocations (blue spots) and a central grain boundary in green ${ }^{17}$. The smectic layers are represented for a $8 \mathrm{CB}$ thin film with typical thickness of $230 \mathrm{~nm}$.

We have used arrays of oriented smectic defects of 8CB (4'-octyl-4-biphenylcarbonitrile) thin films deposited on rubbed poly(vinyl alcohol) (PVA) surfaces, the so-called smectic oily streaks ${ }^{19}$. Due to hybrid anchoring at the two interfaces (air/8CB and 8CB/PVA, respectively), the smectic layers become curved in flattened hemicylinders perpendicular to the anchoring on the substrate, itself defined by the rubbing of the substrate (Figure 1). ${ }^{19-21}$ These flattened hemicylinders, with typical periodicity of several hundreds of nanometers, can be detected by polarized optical microscopy (POM) between crossed polarizers, leading to the observation of parallel stripes (Figure 1a). Their internal structure has been determined using combined X-ray diffraction and ellipsometry measurements (Figure 1b). ${ }^{17}$ It is associated with the presence of two rotating grain boundaries per hemicylinder (in red in Figure 1b). Along each rotating grain boundary, three edge dislocations are expected (in blue in Figure 1b), in relation with a different number of curved and flat smectic layers from each part of the rotating grain boundary. For one given hemicylinder, this leads to six edge dislo- 
cations, all being parallel to each other (i.e., oriented along the OX direction, perpendicular to the substrate rubbing, parallel to the hemicylinder axis and thus parallel to the stripes visible by POM - Figure 1) and separated from each other by a distance of approximately $50 \mathrm{~nm}$. It has been shown that the rotating grain boundary profile does not depend on the thickness of the smectic film. ${ }^{17}$ This consequently leads to a constant number of 6 1D-linear topological defects per hemicylinder. A sublayer made of smectic layers perpendicular to the substrate has been also revealed, allowing to preserve the planar unidirectional anchoring on the PVA surface. A central defect is thus induced (green line in Figure 1b), directly on top of the sublayer. It possibly corresponds to a locally melted $2 \mathrm{D}$ area, thus forming a $2 \mathrm{D}$ ribbon-like topological defect per hemicylinder, of width around 400nm. ${ }^{17}$

Composite films (Oily streaks of $8 \mathrm{CB}$ containing gold nanoparticles) have been obtained (see section 'Materials and Methods'). Different diameters of nanoparticles were investigated : $D=5,6,7$, and $8.3 \mathrm{~nm}$, with a specific focus on the diameter $D=6 \mathrm{~nm}$ (Figure SI2). The latter NPs are covered by dodecanethiol ligands. We tuned the average concentration of NPs, $c_{\text {avg }}$, in the $8 \mathrm{CB}$ films between 700 and $2500 \mathrm{NPs} \mu \mathrm{m}^{-2}$. These surface coverages were determined from Rutherford Back Scattering (RBS) measurements (see section 'Materials and Methods'). For $c_{\text {avg }}$ between 700 and $1300 \mathrm{NPs} \mu \mathrm{m}^{-2}$, we have identified by POM two distinct structures: either the normal oily streaks, corresponding to well-dispersed NPs (Figure 2a); or locally more densely concentrated domains of NPs forming superstructures superimposed onto the normal oily streak pictures (Figure $2 b$ ). For both structures, the oily streak texture observed by POM between crossed polarizers appears only slightly modified with respect to pure $8 \mathrm{CB}$ (Figure SI3). The two different kinds of NP localization appeared to be difficult to control in relation with the solvent evaporation process. However, for average concentrations above $c_{\text {avg }}=1300 \mathrm{NPs} \mu \mathrm{m}^{-2}$, only the second kind of texture was observed (Figure SI3).

Firstly focusing on the well-dispersed NPs (Figure 2a), Figure 2c shows a typical extinction spectrum measured right in the center of Figure 2a (probed area of $40 \times 40 \mu \mathrm{m}^{2}$ ) 

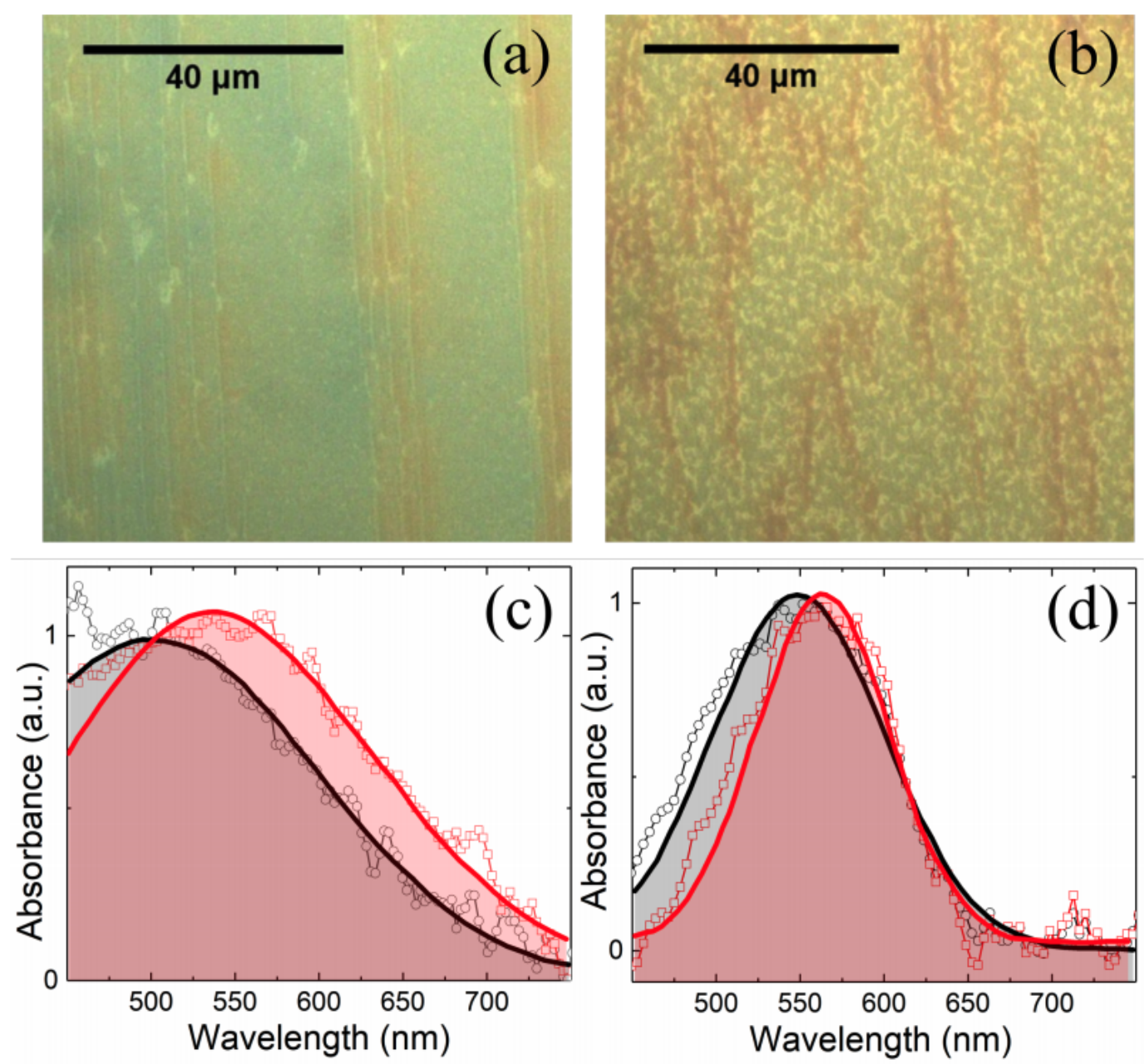

Figure 2: (a) POM picture with parallel polarizers for an area of well-dispersed NPs of diameter $D=6 \mathrm{~nm}$. (b) POM picture with parallel polarizers for an area where the NPs (diameter $D=6 \mathrm{~nm}$ ) form locally denser domains. (c) Normalized extinction spectrum measured in the center of Figure 2a with superimposed gaussian fits for the determination of the resonance wavelength (see Materials and Methods). In black is the extinction for light polarization perpendicular to the $8 \mathrm{CB}$ oily streak stripes, in red for the parallel polarization; (d) Normalized extinction spectrum measured in the center of Figure 2b.

displaying the LSP resonance of the gold NPs with a diameter $D=6 \mathrm{~nm}$ embedded in the $8 \mathrm{CB}$ oily streaks. For a polarization of the incident light perpendicular to the oily streaks (black spectrum), the extinction wavelength is slightly smaller than the one of NPs dispersed in toluene (Figure SI2, $\lambda_{o}=516 \mathrm{~nm}$ ), with $\lambda_{\perp}=500 \mathrm{~nm}$. This confirms previous findings showing that the optical index for LSP resonance is dominated by the dodecanethiol ligands grafted around the NPs. ${ }^{22,23}$ Otherwise a red-shift of the LSP resonance would have been 
observed. In contrast, a clear red-shift of the LSP resonance is observed for a parallel polarization, with $\lambda_{\|}=550 \mathrm{~nm}$. This phenomenon is not due to the $8 \mathrm{CB}$ birefringence $\left(\mathrm{n}_{o}=\right.$ $\left.1.52, \mathrm{n}_{e}=1.67\right)$ since the $8 \mathrm{CB}$ variation of optical index with polarization in relation with oily streak geometry would have induced a blue-shift of the LSP resonance. The observed red-shift indicates that a significant coupling between NPs occurs only in the direction parallel to the oily streak dislocations, in relation with the formation of chains all parallel from each other and parallel to the dislocations. ${ }^{23,24}$ All the extinction measurements for the welldispersed NPs with $D=6 \mathrm{~nm}$ are represented with blue stars in the graph of $\lambda_{\|}$as a function of $\lambda_{\perp}$ (Figure 3). It appears that while $\lambda_{\perp}$ varies only little from one area to another, i.e. the NPs remain individual in this direction, $\lambda_{\|}$changes substantially, with a maximum for $\lambda_{\|}$equal to $562 \mathrm{~nm}$. This shows that only chains parallel to the LC linear defects are formed, the longer chains being associated with $\lambda_{\|}=562 \mathrm{~nm}$. Secondly focusing on the zones with locally dense domains (Figure 2b), the optical properties of the NPs differ from the zones with well-dispersed NPs (Figures $2 \mathrm{c}$ and $2 \mathrm{~d}$ ). On Figure $2 \mathrm{~d}, \lambda_{\|}$is still largely red-shifted, but now $\lambda_{\perp}$ also appears red-shifted with respect to the LSP band wavelength of NPs in toluene (Figure SI2). This indicates that a coupling between NPs also occurs in the direction perpendicular to the smectic oily streaks. However $\lambda_{\perp}$ is still smaller than $\lambda_{\|}$. Figure 3 displays in yellow and purple the corresponding values of $\left(\lambda_{\perp}, \lambda_{\|}\right)$for each measured zone. Each extinction graph associated with the yellow and purple spots has been fitted by a single gaussian curve to obtain $\lambda_{\perp}$ and $\lambda_{\|}$mean values. It shows that $\lambda_{\perp}$ regularly increases but remains always smaller than $\lambda_{\|}$, finally reaching a maximum corresponding to $\lambda_{\perp} \approx \lambda_{\|} \approx 580$ $\mathrm{nm}$ (purple spot), associated with the densest and/or largest NP organizations.

In order to gain more insight on the second part of the diagram drawn in Figure 3, we have analyzed the sample corresponding to the purple dots displayed in Figure 3 by grazing incidence small-Angle X-ray scattering (GISAXS) and transmission small-Angle X-ray scattering (TSAXS) performed at Soleil synchrotron facility (SIXS beamline). The sample corresponds to $c_{\text {avg }}=2500 \mathrm{NPs} \mu \mathrm{m}^{-2}$ (RBS measurements), with POM pictures displaying 


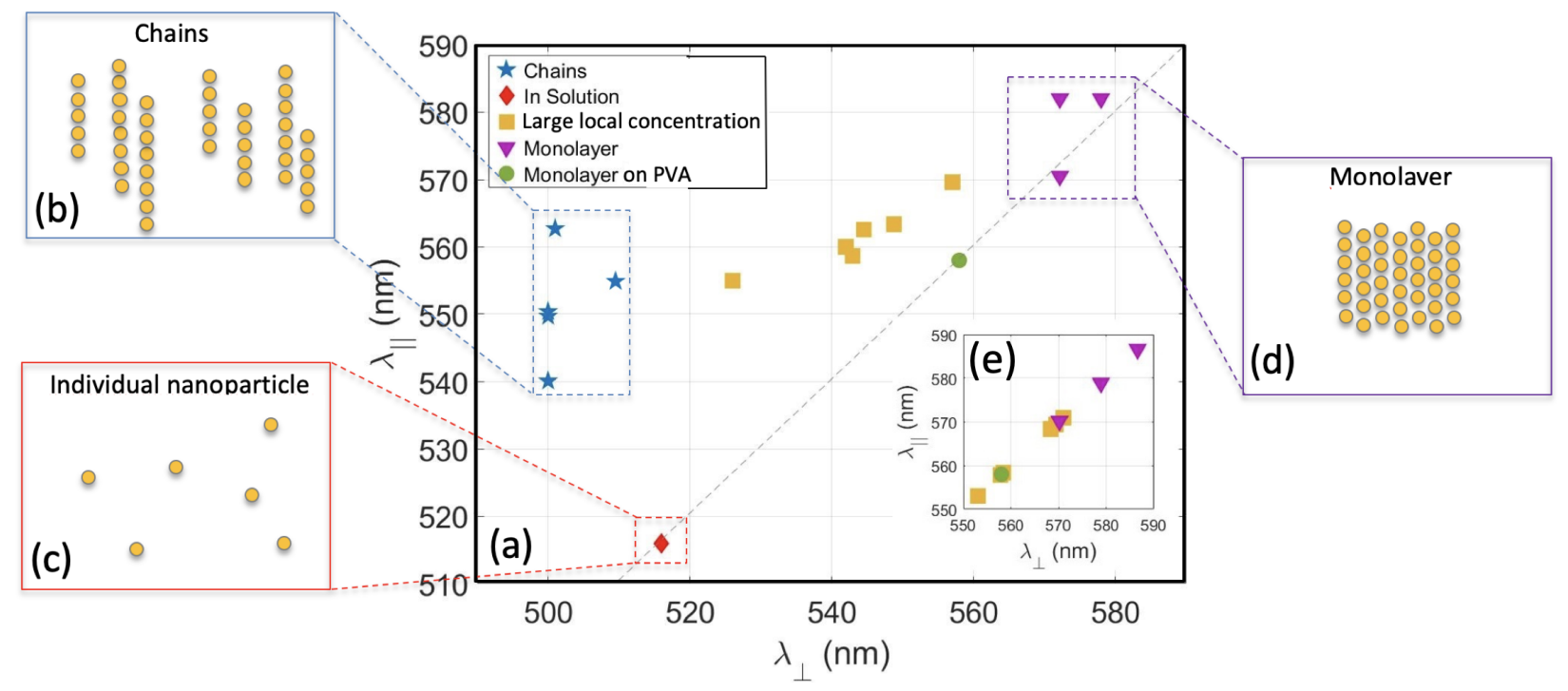

Figure 3: (a) Summary of the extinction measurements represented by the $\left(\lambda_{\perp}, \lambda_{\|}\right)$values, obtained for NPs with diameter $D=6 \mathrm{~nm}$. (b) In blue are shown the measurements for chains, corresponding to areas with well-dispersed NPs as seen by POM. (c) In red is presented the measurement for isolated nanoparticles in solution. (d) In yellow and purple are shown the measurements, corresponding only to areas where dense domains of NPs are identified by POM. Specifically the measurements performed on the sample with the highest NP concentration are shown in purple. In green is the result obtained for a monolayer of NPs without $\mathrm{LC}\left(\lambda_{\perp}=\lambda_{\|}=558 \mathrm{~nm}\right)$. The dotted line is of slope 1, corresponding to isotropic optical properties $\left(\lambda_{\perp}=\lambda_{\|}\right)$. (e) the same data differently analyzed : the extinction of the "long chains" (blue spot of largest $\lambda_{\|}$) has been subtracted from the extinction of the yellow and purple spots to lead to isotropic extinction, of $\lambda$ values again shown in yellow and purple.

the presence of dense NP domains, similar to Figure 2b (Figure SI3). Figure 4b shows the GISAXS pattern collected with the X-ray beam parallel to the 8CB stripes (see 'Materials and methods') while GISAXS data collected with the same NPs without 8CB (ie. just deposited on rubbed PVA) are presented in Figure 4a. We will denote by $\mathbf{q}$ the scattering vector, $q_{z}$ its component normal to the substrate, $q_{\|}$its in-plane component parallel to the oily streaks and $q_{\perp}$ its in-plane component perpendicular to the oily streaks. In Figure 4a, three symmetric pairs of rods are visible with their respective $q_{\perp}$ positions being in the ratio 1, $\sqrt{3}, 2$. This result indicates that the spherical NPs exhibit a planar hexagonal organization, as usually observed when NPs are directly deposited from the solution on a substrate. This is thus in agreement with observations carried out by scanning electron microscopy (Figure SI4). The presence of well-defined rods indicates a good quality of the NPs in terms 


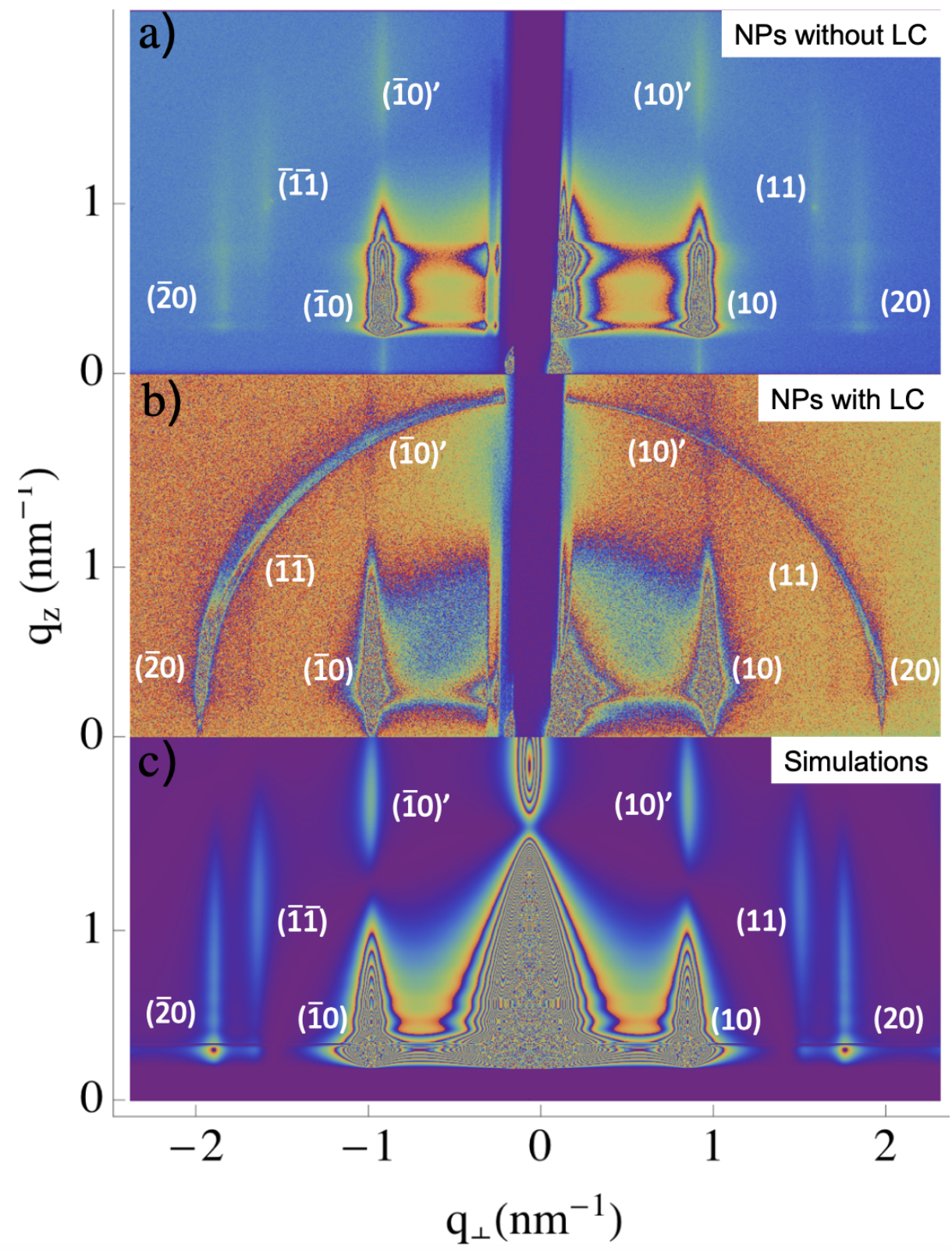

Figure 4: (a) GISAXS pattern of a sample with NPs deposited on a rubbed polymer substrate without LC, with the various rods of the hexagonal NP assembly being indicated. (b) GIXAXS pattern for a sample of average NP concentration of $c_{a v g}=2500 \mathrm{NPs} \mu \mathrm{m}^{-2}$, collected with the X-ray beam parallel to the stripes, where the semi-circle corresponds to the hemicylindrical stacking of the smectic layers and the various scattering rods correspond to the signal of the hexagonal NP assembly. (c) Simulated GISAXS pattern for a planar hexagonal network of spheres $(D=6 \mathrm{~nm})$ supported on a PVA/glass substrate. For the sake of simplicity, the intensity map is the sum of two simulations corresponding to $q_{10}$ parallel to $q_{\perp}$ and to $q_{11}$ parallel to $q_{\perp}$. The DWBA calculations were done using the FitGISAXS software package ${ }^{25}$. 
of polydispersity (size and shape). The three pairs of rods correspond to the $((10),(\overline{1} 0))$

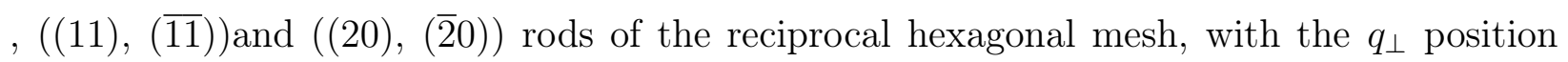
of the (10) rod being $q_{(10)}=0.92 \mathrm{~nm}^{-1}$. At large $q_{z}$, the different characteristic features of the different rods can be unambiguously attributed to the form factor of a dense sphere, $F(q)=4 \pi \frac{\sin (q R)-q R \cos (q R)}{q^{3}}$. The (10) rod profile exhibits two detectable maxima, (10) and $(10)^{\prime}$, in contrast with the (11) rod, whose first maximum appears at a larger $q_{z}$ value than for the (10) and (20) rods. This form factor effect is clearly evidenced in Figure 4c, which shows the GISAXS intensity calculated in the DWBA using the FitGISAXS package ${ }^{25}$ for a planar hexagonal network of spheres $(D=6 \mathrm{~nm})$ supported on a PVA/glass substrate. The main difference between the experimental and simulated patterns lies in the shape of the (10) rod at $q_{z} \approx 0.7 \mathrm{~nm}^{-1}$ that suggests the presence of some multilayers coexisting with monolayers in the sample without LC.

In Figure $4 \mathrm{~b}$, a half-circle with radius $q_{L C} \approx 2 \mathrm{~nm}^{-1}$ appears. It corresponds to the scattering of the $8 \mathrm{CB}$ smectic layers, stacked on top of each other and forming hemicylindrical structures (Figure 1) ${ }^{17}$. Although this half-circle almost prevents the observation of the (20) and $(\overline{2} 0)$ rods, the $((10),(\overline{1} 0))$ and $((11),(\overline{1} 1))$ rods are discernable at the same $q_{z}$ position as without $8 \mathrm{CB}$, their $q_{\perp}$ positions being in the ratio $1, \sqrt{3}$, with $q_{(10)}=0.99 \mathrm{~nm}^{-1}$. At large $q_{z}$ around $1.7 \mathrm{~nm}^{-1}$, the $(10)^{\prime}$ signal is also clearly visible, with the same intensity variation along $q_{z}$ with respect to the (10) than without $8 \mathrm{CB}$ (see Figure SI5). The presence of vertical rods and the similarity of their profile above the horizon with the one of Figure 4c demonstrate that in $8 \mathrm{CB}$ also the NPs are arranged in a planar 2D hexagonal network of spheres parallel to the substrate. The (10) rod on Figure $4 \mathrm{~b}$ has a shape very close to the one of Figure 4c, without any bump at $q_{z} \approx 0.7 \mathrm{~nm}^{-1}$, in contrast with Figure 4a. The presence of this bump at $q_{z} \approx 0.7 \mathrm{~nm}^{-1}$ was observed in only 3 areas over 10 , meaning that in $8 \mathrm{CB}$, monolayers are preferentially formed rather than multilayers. The (10) rod has been analyzed in addition through TSAXS measurements with the sample perpendicular to the X-ray beam ('Materials and methods'). The obtained scattered circle (Figure 5a) shows that the NP domains are 
made of hexagonal meshes of NPs presenting a large distribution of possible crystallographic orientations (2D powder). However a careful analysis of the scattered intensity along this circle reveals the presence of an intensity modulation with a $60^{\circ}$ period and maxima of scattered intensity at $0^{\circ}, 60^{\circ}, 120^{\circ}$ and $180^{\circ}$ (Figure 5b). This confirms the hexagonal structure of the NP networks in $8 \mathrm{CB}$. A preferred orientation of the hexagonal meshes in $8 \mathrm{CB}$ can be deduced in contrast with the NP network without LC (Figure SI6). The dense direction of the NP network, the $\langle 10\rangle$ direction, is preferably parallel to the oily streaks, in other words parallel to the chain direction observed at smaller concentration and parallel to the LC dislocations. In agreement with the comparison between Figure $4 \mathrm{~b}$ and Figure 4a, this favorable orientation observed in LC leads to a smaller intensity of the (11) rods in GISAXS measurements in LC (Figure 4b) with respect to the case without LC (Figure 4a). The $q_{\perp}$ position of the (10) rod in $8 \mathrm{CB} q_{(10)}=0.99 \mathrm{~nm}^{-1}$ corresponds to an average gap between NPs equal to $s=4 \times \pi \times \sqrt{3} / q_{(10)}-D=1.34 \pm 0.05 \mathrm{~nm}$, after averaging over 12 areas of the sample. For the NP hexagonal network on rubbed PVA without LC $\left(q_{(10)}=0.92 \mathrm{~nm}^{-1}\right)$, a gap of $s=1.93 \mathrm{~nm} \pm 0.05 \mathrm{~nm}$ can be extracted as averaged over 5 zones. This value is very close to the one previously obtained for similar NP hexagonal networks with NPs of diameter $D=6 \mathrm{~nm}$, also covered by dodecanethiol ${ }^{26,27}$. This demonstrates a LC-induced shortening of the inter-NP gap in the hexagonal network of NPs in LC with respect to hexagonal networks without $8 \mathrm{CB}$. This is consistent with the largest LSP resonance wavelength for the purple dots on Figure 3 with respect to the green dot associated with hexagonal networks without LC on PVA substrates.

Our analysis reveals that in oily streaks, for NPs well-dispersed in small enough amount, chains of NPs are formed with NPs of diameter $6 \mathrm{~nm}$, but also with NPs of diameter $5 \mathrm{~nm}, 7$ $\mathrm{nm}$ and $8.3 \mathrm{~nm}$ (see Figure SI7). While it had been demonstrated that non distorted smectic films could avoid any aggregation of similar gold $\mathrm{NPs}^{28}$, for distorted smectic films, the role of linear defect cores had been previously highlighted: Nanorods are aligned in oily streaks perpendicular to the LC director but parallel to the defect $\operatorname{cores}^{29,30}$. This demonstrates 
a)

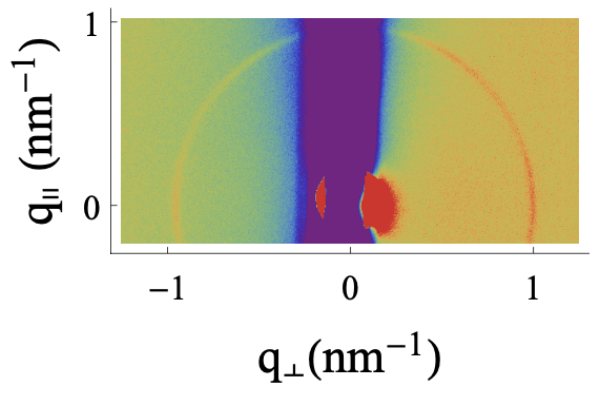

b)

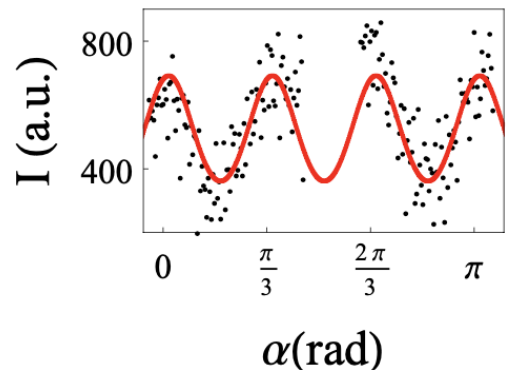

Figure 5: (a) TSAXS pattern of the same sample as in Figure 4b, observed in transmission with the X-ray beam perpendicular to the sample. The circle corresponds to the (10) rod visible in Figure 4b and demonstrates the possible orientations of the hexagonal crystals in the NP domains (b) Modulation of the intensity along the circle extracted after careful removal of the background. The angle $\alpha$ corresponds to the various positions along the circle shown in (a), the 0 and $180^{\circ}$ values corresponding to the horizontal, defined as parallel to the $8 \mathrm{CB}$ dislocations.

a trapping phenomenon by the linear defect cores. Trapping of spherical NPs leading to formation of NP chains has been observed first with smaller NPs with diameter of $4 \mathrm{~nm}^{23,24}$. We now extend this result to larger NP diameters ( $5-8.3 \mathrm{~nm}$ of diameter), suggesting an efficient trapping of the NPs by these linear defect cores for an extended range of NP size. Formation of NP chains of highly anisotropic optical extinction can occur for larger NP diameters thanks to the large enough distance between neighboring dislocations of the order of $50 \mathrm{~nm}$. The question now is how many NPs can form chains without aggregation outside the cores ? RBS maximum average concentration of NPs (diameter $D=6 \mathrm{~nm}$ ) leading to NP chains is equal to $\mathrm{c}_{\text {avg }}=1300 \mathrm{NPs} \mu \mathrm{m}^{-2}$. Assuming an inter-NP distance of $D+1.3 \approx 7.4$ $\mathrm{nm}, 1.3 \mathrm{~nm}$ being the inter-NP gap found in the NP hexagonal networks in LC, expected to be not fundamentally different from the one in the NP chains, it can be calculated that a linear defect of length $1 \mu \mathrm{m}$ contains up to approximately 140 NPs. As a result, for the LC thickness of $170 \mathrm{~nm}$ of the sample with $\mathrm{c}_{\text {avg }}=1300 \mathrm{NPs} \mu \mathrm{m}^{-2}$, the period of oily streak hemicylinders ${ }^{17}$ is of the order of $550 \mathrm{~nm}$ and the critical concentration necessary to entirely fill the 6 dislocations is : $c_{c r i t} \approx 1530 \mathrm{NPs} \mu \mathrm{m}^{-2}$. $1300 \mathrm{NPs} \mu \mathrm{m}^{-2}$ corresponds to $85 \%$ of 
the 6 linear dislocations shown in Figure 1 being filled by NPs. This demonstrates that the smectic defects cores can be almost fully filled with no 3D aggregation outside the core, also suggesting formation of particularly long NP chains. Are thus the dislocations also almost fully filled for the samples displaying dense domains that appear yellow in POM pictures under parallel polarizers (Figure 2b and SI3)? The inhomogeneous POM contrast suggests an inhomogeneous NP concentration. This suggests a locally larger NP concentration than the one revealed by average RBS measurements that are performed over a large area of roughly $0.2 \mathrm{~mm}^{2}$. RBS measurements of the the sample studied by GISAXS and TSAXS (Figure 4 and 5) leads to $c_{a v g}=2500 \mathrm{NPs} \mu \mathrm{m}^{-2}$. It is larger than $\mathrm{c}_{\text {crit }}$ which must be equal to $1050 \mathrm{NPs} \mu \mathrm{m}^{-2}$ for this sample of average thickness around $250 \mathrm{~nm}$ and oily streak hemicylinder period of the order of $650 \mathrm{~nm}$. This definitely shows that in the dense NP domains, the LC dislocations can be fully filled by NPs for this very concentrated sample. For the sample of Figure 2b, the RBS measurements yield an average NP density of only 820 NPs $\mu \mathrm{m}^{-2}$, smaller than $\mathrm{c}_{\text {crit }}=1050 \mathrm{NPs} \mu \mathrm{m}^{-2}$ for this sample of average thickness around $250 \mathrm{~nm}$. This suggests that NP chains may not be formed everywhere on this sample. We can consider that no or only few NPs are present out of the dense domains. POM analysis leads to $16 \%$ of the surface being covered with NP domains. This corresponds to a local concentration of $5120 \mathrm{NPs} \mu \mathrm{m}^{-2}$, now larger than $\mathrm{c}_{\text {crit }}=1050 \mathrm{NPs} \mu \mathrm{m}^{-2}$. From the sample of Figure 2b-d (yellow spot on Figure 3) to the sample of Figure 4-5 (purple spot on Figure 3), the average and local NP concentration increase but the local NP concentration remains always larger than $c_{\text {crit }}$. Figure 3 can thus be associated with a phase diagram controlled by NP concentration. The blue spots correspond to the smallest concentration for which only chains are formed. The yellow and purple spots correspond to local NP concentrations larger than the necessary amount of NP to fully fill the linear dislocations. X-ray results show the presence of 2D hexagonal monolayers that could coexist with 1D linear NP chains. This would explain the small remaining anisotropy of the optical absorption associated with yellow and purple spots. In order to further test this hypothesis, we have subtracted from 
the extinction shown in Figure 2d the extinction of long chains $\left(\lambda_{\perp}=500 \mathrm{~nm}, \lambda_{\|}=562 \mathrm{~nm}\right)$ without normalization and with the use of a multiplying factor of 0.48 . This factor takes into account the not full coverage of NPs on the surface already suggested by Figure $2 \mathrm{~b}$. A quasi isotropic extinction is obtained at $\lambda=558 \mathrm{~nm}$ (Figure SI8). It is consistent with coexisting long chains and 2D monolayer patches with the assumption that the anisotropy of the surrounding $8 \mathrm{CB}$ matrix is of negligible influence ${ }^{22,23,31}$. This fitting procedure can be performed for each blue or orange spot shown on Figure 3. The obtained values for the $\lambda$ of the hexagonal monolayer patches are shown in Figure 3e. $\lambda$ increases from $553 \mathrm{~nm}$ to $586 \mathrm{~nm}$ when the local concentration increases. This may correspond to decreasing inter-NP gaps or/and increasing sizes of the hexagonal networks when the local concentration increases. The question to solve now concerns the origin of these 2D NP hexagonal networks, their shape and localization in the 8CB matrix.

It is known that the dodecanethiol ligands grafted on gold substrates mostly induce tilted or homeotropic anchoring of $\mathrm{LCs}^{32,33}$. We thus expect that if the NPs would be directly deposited on top of the substrate, they would destroy the 8CB oily streaks, which is not observed. In contrast, in presence of the dense NP domains, we observed clear GISAXS rings associated with the smectic layer structure of the oily streaks (Figure 4b). Moreover POM pictures between cross polarizers show the oily streak stripes everywhere including on top of the domains (Figure SI3). These POM observations also demonstrate that the NP domains do not sit at the $8 \mathrm{CB} /$ air interface. Otherwise they would lead to a decrease of the POM contrast between crossed polarizer which is not observed. The fact that dense NP domains do not sit at the $8 \mathrm{CB} /$ air interface is confirmed by a careful analysis of the RBS signal of the $\mathrm{Au}$ atoms in the $8 \mathrm{CB}$ film. A signal of larger width for the samples with dense NP domains with respect to the ones where only NP chains are formed is observed (Figure SI9). This is linked with a large number of Au atoms deeply embedded in the LC film in presence of NP domains, that are not observed when only chains are formed in the LC film. The only interpretation for these experimental results is thus the following: The 2D NP networks are localized elsewhere 
than in and around the LC dislocations. They are more deeply embedded in the LC film and must be confined in the planar 2D ribbon-like defects of each oily streak hemicylinder (green line in Figure 1). The 2D ribbon-like shape of this central defect, parallel to the substrate, of width around $400 \mathrm{~nm}$ may induce a 2D confinement leading to the observed NP planar hexagonal networks. The confinement of fluorescent NPs in these 2D defects at large concentration should then lead to the formation of fluorescent ribbons localized in the center of the smectic hemicylinders. This assumption is perfectly confirmed by the observed ribbon-like shape of fluorescent domains made of fluorescent nanorods (CdSe/CdS dot-in-rods of length $20 \mathrm{~nm}$ and diameter $7 \mathrm{~nm}$, coated with phosphonic acid $\left.{ }^{34}\right)$. They have been observed by fluorescent microscopy at large NP concentration in $8 \mathrm{CB}$ oily streaks, revealing in addition a specific localization in the center of the $8 \mathrm{CB}$ hemicylinders (Figure SI10). This is in contrast with the gold nanorod localization observed previously at small concentration. These latter ones were shown to form at small concentration a large majority of aligned nanorod chains, thus trapped in the smectic dislocations and not in the 2D-ribbon like topological defect. This not only confirms that the 2D NP networks are localized in the central 2D ribbon-like topological defects but also demonstrates that nanorods behave like nanospheres with their localization in the smectic oily streak topological defects being driven by the concentration. The interpretation of Figure 3 as a phase diagram driven by the NP local concentration becomes particularly clear: A formation of NP chains (in blue in Figure 3) in the linear dislocations occurs first at small NP concentration with the length/number of the NP chains increasing with NP concentration until the dislocations are quasi-filled. At larger concentration, formation of $2 \mathrm{D}$ hexagonal networks follows in the $2 \mathrm{D}$ ribbon-like topological defects, coexisting with NP chains (in yellow-purple in Figure 3). They would become larger and possibly of larger amount when the local concentration still increases. These results show that formation of the NP chains in the linear dislocation cores is more favorable than the NP $2 \mathrm{D}$ confinement. They also demonstrate that 2D topological defects induce a 2D confinement that ultimately leads to formation of planar 2D NP hexagonal networks. The NP hexagonal 
networks can be oriented along a single direction driven by the 2D topological defect, parallel to the oily streak direction. This can be explained by $2 \mathrm{D}$ hexagonal monolayers preferably formed at the border of the central defect, at the basis of the rotating grain boundary (see Figure 1), which is parallel to the oily streaks. The 2D ribbon-like topological defect however only partially orients the trapped hexagonal network as shown by the structured circle of Figure 5. When the NP concentration increases some monolayer patches could grow far from the central defect border and would become disoriented. This suggests an increase of disorder when the concentration increases, indeed observed in the hexagonal networks of small NPs of diameter $4 \mathrm{~nm}$ (Figure SI11). It would be now interesting to establish the critical concentration able to induce a large majority of well-oriented 2D NP networks. The enlargement of this feature to oriented 2D networks of nanorods could lead to formation of highly anisotropic 2D networks of nanorods. With gold nanorods, highly anisotropic LSP resonance would be expected, firstly due to nanorods all parallel from each other ${ }^{35}$, secondly due to highly anisotropic electromagnetic coupling between nanorods.

In conclusion, we have shown how two different kinds of confinement induced by different topological defects lead to two different kinds of gold nano-sphere organizations. In the smectic oily streaks made of oriented arrays of 1D dislocations and 2D ribbon-like grain boundaries, we show that the dislocations induce formation of oriented NP chains, whereas the ribbon-like grain boundaries induce formation of planar 2D hexagonal networks of NPs. The efficiency of the 1D dislocations for the trapping of NPs is the highest. They can be filled at $85 \%$ by NPs without any significant aggregation outside the defect cores, allowing for the formation of a large number of long NP chains, all oriented parallel from each other, along a single direction defined by the rubbed polymer substrate. The $2 \mathrm{D}$ confinement also allows for the transmission of the anisotropy of the overall structure through a favorable orientation of the hexagonal network, the $\langle 10\rangle$ direction being more favorably oriented parallel to the defect orientation, possibly due to the orienting influence of the border of the ribbon-like grain boundaries. Owing to the excellent large-scale order of these defect cores, not only the 
NP chains but also the NP hexagonal networks can be oriented along the desired direction, suggesting a possible new route for the creation of either highly anisotropic 1D or oriented 2D NP networks. In addition these results demonstrate that hierarchical structure of topological defects can be used to sequentially build different kinds of NP assemblies controlled by the NP concentration. This leads to the establishment of a phase diagram for the NP organization driven by NP concentration. Rich perspectives are then opened based on the possible creation in hierarchical structures of LC topological defects of coexisting NP assemblies of different kinds, localized in different confining areas of a same LC film that are however close from each other. They could thus interact thanks to their proximity but also taking advantage of the soft and activable character of the surrounding LC matrix.

\section{ASSOCIATED CONTENT}

The Supporting Information is available free of charge on the at

Details about nanoparticles synthesis and characterization, simple preparation, X-ray and Rutherford backscattering spectroscopy (PDF).

\section{Acknowledgement}

We thank D. Limagne, C. Rafaillac and B. Voisin for technical supports ; D. Demaille and L. Becerra for the electronic Microscopy ; Y. Vickridge for the analysis of the RBS data. We thank CNES (Centre National d'Etudes Spatiales) for supporting this project. Concerning the financial funding, S.P. Do, A. Missaoui and E. Lacaze thank the French state funds managed by the ANR within the Investissements d'Avenir programme under reference ANR11-IDEX-0004-02, and more specifically within the framework of the Cluster of Excellence MATISSE. B. Donnio and J-L. Gallani thank the CNRS and the University of Strasbourg for support. We also thank Synchrotron SOLEIL for providing the beam on SixS beamline. 


\section{References}

(1) Chuang Isaac; Durrer Ruth; Turok Neim; Yurke Bernard, B. Cosmology in the Laboratory: Defect Dynamics in Liquid Crystals. Science 1991, 251, 1336-1342.

(2) Kibble Tom, T. Phase-Transition Dynamics in the Lab and the Universe. Physics Today 2007, 60 .

(3) Ball John Macleod; Zarnescu Arghir, A. Orientability and Energy Minimization in Liquid Crystal Models. Archive for Rational Mechanics and Analysis 2011, 202, 493535.

(4) Del Campo Adolfo; Zurek Wojciech H, H. Universality of phase transition dynamics: Topological defects from symmetry breaking. International Journal of Modern Physics A 2014, 29, 1430018.

(5) Hiroyuki Yoshida; Yuma Tanaka; Kosuke Kawamoto; Hitoshi Kubo; Tetsuya Tsuda; Akihiko Fujii; Susumu Kuwabata; Hirotsugu Kikuchi; Masanori Ozaki, O. Nanoparticlestabilized cholesteric blue phases. Applied Physics Express 2009, 2.

(6) Karatairi Eva; Rožic Brigita; Kutnjak Zdravko; Tzitzios Vassilios; Nounesis George; Cordoyiannis George; Thoen Jan; Glorieux Christ; Kralj Samo, S. Nanoparticle-induced widening of the temperature range of liquid-crystalline blue phases. Phys. Rev. E 2010, 81, 041703.

(7) Rozic Brigita; Tzitzios Vasileios; Karatairi Eva; Tkalec Uros; Nounesis G; Kutnjak Zdravko; Cordoyiannis G; Rosso Riccardo; Virga Epifanio; Musevic I; Kralj Samo, O. Theoretical and experimental study of the nanoparticle-driven blue phase stabilisation. The European physical journal. E, Soft matter 2011, 34, 1-11.

(8) Senyuk Bohdan; Evans Julian S; Ackerman Paul J; Lee Taewoo; Manna Pramit; Vigderman Leonid; Zubarev Eugene R; van de Lagemaat Jao; Smalyukh Ivan I, I. Shape- 
Dependent Oriented Trapping and Scaffolding of Plasmonic Nanoparticles by Topological Defects for Self-Assembly of Colloidal Dimers in Liquid Crystals. Nano Letters 2012, 12, 955-963.

(9) Cordoyiannis George; Jampani Venkata; Kralj Samo; Dhara Surajit; Tzitzios Vasileios; Basina Georgia; Nounesis George; Kutnjak Zdravko; Tripathi Chandra Shekhar Pati; Losada-Perez Patricia; Jesenek Dalija; Glorieux Christ; Musevic Igor; Zidansek Aleksander; Amenitsch Heinz; Thoen Jan, J. Different modulated structures of topological defects stabilized by adaptive targeting nanoparticles. Soft Matter 2013,

(10) Evans Julian S; Ackerman Paul J; Broer Dirk J; van de Lagemaat Jao; Smalyukh Ivan I, I. Optical generation, templating, and polymerization of three-dimensional arrays of liquid-crystal defects decorated by plasmonic nanoparticles. Phys. Rev. E 2013, 87, 032503.

(11) Gharbi Mohamed Amine; Manet Sabine; Lhermitte Julien; Brown Sarah; Milette Jonathan; Toader Violeta; Sutton Mark; Reven Linda, L. Reversible Nanoparticle Cubic Lattices in Blue Phase Liquid Crystals. ACS Nano 2016, 10, 3410-3415, PMID: 26900753.

(12) Mundoor Haridas; Sheetah Ghadah H; Park Sungoh; Ackerman Paul J; Smalyukh Ivan I; van de Lagemaat Jao, J. Tuning and Switching a Plasmonic Quantum Dot Sandwich in a Nematic Line Defect. ACS Nano 2018, 12, 2580-2590, PMID: 29489324.

(13) Lopez-Leon Teresa; Fernandez-Nieves Alberto, A. Drops and shells of liquid crystal. Colloid and Polymer Science 2011, 289, 345-359.

(14) Lopez-Leon Teresa; Koning Vinzenz; Devaiah K; Vitelli Vincenzo; Fernandez-Nieves Alberto, A. Frustrated nematic order in spherical geometries. Nature Physics 2011, 7, $391-394$. 
(15) Sec David; Porenta Tine; Ravnik Miha; Zumer Slobodan, S. Geometrical frustration of chiral ordering in cholesteric droplets. Soft Matter 2012, 8, 11982-11988.

(16) Darmon Alexandre; Benzaquen Michael; Dauchot Olivier; Lopez-Leon Teresa, T. Waltzing route toward double-helix formation in cholesteric shells. Proceedings of the National Academy of Sciences 2015, 113.

(17) Coursault Delphine; Zappone Bruno; Coati Alessandro; Boulaoued Athmane; Pelliser Laurent; Limagne Denis; Boudet Nathalie; Ibrahim Bicher Haj; de Martino Antonello; Alba Michel; Goldmann Michel; Garreau Yves; Gallas Bruno; Lacaze Emmanuelle, E. Self-organized arrays of dislocations in thin smectic liquid crystal films. Soft Matter 2016, 12, 678-688.

(18) Nemitz Ian; Gryn Iryna; Boudet Nathalie; Lemieux Robert; Goldmann Micheel; Zappone B; Petschek R; Rosenblatt Charles; Lacaze Emmanuelle, E. Observations of a streak texture in the hybrid-aligned smectic-C phase. Soft Matter 2018, 14.

(19) Michel Jean-Philippe; Lacaze Emmanuelle; Goldmann Michel; Gailhanou Marc; de Boissieu Marc; Alba Michel, M. Structure of Smectic Defect Cores: X-Ray Study of 8CB Liquid Crystal Ultrathin Films. Physical Review Letter 2006, 96, 0278031-0278034.

(20) Michel Jean-Philippe; Lacaze Emmanuelle; Goldmann Michel; Gailhanou Marc; Boissieu Marc; Alba Michel, M. Structure of Smectic Defect Cores: X-Ray Study of 8CB Liquid Crystal Ultrathin Films. Physical review letters 2006, 96, 027803.

(21) Zappone Bruno; Lacaze Emmanuelle, E. Surface-frustrated periodic textures of smecticA liquid crystals on crystalline surfaces. Phys. Rev. E 2008, 78, 061704.

(22) Mulvaney, P. Surface Plasmon Spectroscopy of Nanosized Metal Particles. Langmuir 1996, 12, 788-800. 
(23) Coursault Delphine; Blach Jean-Francois; Grand Johan; Coati Alessandro; Vlad Alina; Zappone Bruno; Babonneau David; Levi Georges; Felidj Nordin; Donnio Bertrand; Gallani Jean-Louis; Alba Michel; Garreau Yves; Borensztein Yves; Goldmann Michel; Lacaze Emmanuelle, E. Tailoring Anisotropic Interactions between Soft Nanospheres Using Dense Arrays of Smectic Liquid Crystal Edge Dislocations. ACS Nano 2015, 9, $11678-11689$.

(24) Coursault Delphine; Grand Johan; Zappone Bruno; Ayeb Habib; Levi Georges; Felidj Nordin; Lacaze Emmanuelle, E. Linear Self-Assembly of Nanoparticles Within Liquid Crystal Defect Arrays. Advanced Materials 2012, 24, 1461-1465.

(25) Babonneau David, D. FitGISAXS: software package for modelling and analysis of GISAXS data using IGOR Pro. J. Appl. Crystallogr. 2010, 4, 929-936.

(26) Goubet Nicolas; Richardi Johannes; Albouy Pierre-Antoine; Pileni Marie-Paule, M. Which Forces Control Supracrystal Nucleation in Organic Media? Advanced Functional Materials 2011, 21, 2693-2704.

(27) Gauvin Melanie; Wan YanFen; Arfaoui Imad; Pileni Marie-Paule, M. Mechanical Properties of Au Supracrystals Tuned by Flexible Ligand Interactions. The Journal of Physical Chemistry C 2014, 118, 5005-5012.

(28) Pratibha Ramarao; Park Wonhong; Smalyukh Ivan, I. Colloidal gold nanosphere dispersions in smectic liquid crystals and thin nanoparticle-decorated smectic films. Journal of Applied Physics 2010, 10\%, 063511.

(29) Pelliser Laurent; Manceau Mathieu; Lethiec Clotilde; Coursault Delphine; Vezzoli Stefano; Lemenager Godefroy; Coolen Laurent; Devittorio Massimo; Pisanello Ferruccio; Carbone Luigi; Maitre Agnes; Bramati Alberto; Lacaze Emmanuelle, E. Alignment of Rod-Shaped Single-Photon Emitters Driven by Line Defects in Liquid Crystals. Advanced Functional Materials 2015, 
(30) Rozic Brigita; Fresnais Jerome; Molinaro Celine; Calixte Joseph; Umadevi Shivakumar; Lau-Truong Stephanie; Felidj Nordin; Kraus Tobias; Charra Fabrice; Dupuis Vincent; Hegmann Torsten; Fiorini-Debuisschert Celine; Gallas Bruno; Lacaze Emmanuelle, E. Oriented Gold Nanorods and Gold Nanorod Chains within Smectic Liquid Crystal Topological Defects. ACS Nano 2017, 11, 6728-6738.

(31) Sung Yong Parka; D Stroud, D. Splitting of surface plasmon frequencies of metal particles in a nematic liquid crystal. Appl. Phys. Lett. 2004, 85, 2920-2922.

(32) Qi Hao; Hegmann Torsten, T. Multiple Alignment Modes for Nematic Liquid Crystals Doped with Alkylthiol-Capped Gold Nanoparticles. ACS Applied Materials \& Interfaces 2009, 1, 1731-1738.

(33) Christophe Blanc; Delphine Coursault; Emmanuelle Lacaze, E. Ordering nano- and microparticles assemblies with liquid crystals. Liquid Crystals Reviews 2013, 1, 83109.

(34) Carbone Luigi; Nobile Concetta; De Giorgi Milena; Sala Fabio Della; Morello Giovanni; Pompa Pierpaolo; Hytch Martin; Snoeck Etienne; Fiore Angela; Franchini Isabella R.; Nadasan Monica; Silvestre Albert F.; Chiodo Letizia; Kudera Stefan; Cingolani Roberto; Krahne Roman; Manna Liberato, L. Synthesis and Micrometer-Scale Assembly of Colloidal CdSe/CdS Nanorods Prepared by a Seeded Growth Approach. Nano Letters 2007, 7, 2942-2950.

(35) Liu Qingkun; Yuan Ye; Smalyukh Ivan I, I. Electrically and Optically Tunable Plasmonic Guest Host Liquid Crystals with Long-Range Ordered Nanoparticles. Nanoletters 2014, 14, 4071. 